\title{
Evolución en la recuperación de información: de la línea de comandos a la interfaz gráfica Web
}

\author{
José María Angós Ullate \\ Centro de Documentación Científica \\ Universidad de Zaragoza \\ María Jesús Fernández Ruíz \\ Servicio de Información y Gestión Municipal en Internet \\ Ayuntamiento de Zaragoza \\ José Antonio Salvador Oliván \\ Dpto. Ciencias de la Documentación e Historia de la Ciencia \\ Universidad de Zaragoza
}

\subsection{Resumen}

Se analiza el impacto de la interfaz gráfica web sobre las técnicas de planteamiento de la estrategia de búsqueda de información en las bases de datos tradicionales. Se estudian las distintas interfaces gráficas que ofrecen diversos proveedores de información (OCLC, Dialog, STN y BookWhere); las diferencias que ofrecen respecto a las interfaces de línea de órdenes; y diversas estrategias que se pueden utilizar para superar algunos problemas.

Palabras clave: Interfaz gráfica de usuario. World Wide Web. Recuperación de información. Bases de datos. Ecuación de búsqueda. Formularios de búsqueda. Hosts. Distribuidores. OCLC. Dialog. STN. BookWhere.

\subsection{Abstract}

The impact of web-based graphical user interfaces on search strategies is analysed. Several web-based GUI information providers are considered: OCLC, Dialog, STN and BookWhere. The GUIs are compared against command line interfaces. Some techniques to overcome search problems are studied.

Keywords: Graphical user interfaces. World Wide Web. Information retrieval. Databases. Search equation. Search forms. Hosts. Information providers. OCLC. Dialog. STN. BookWhere.

\section{Introducción}

Desde hace algunos años, se están produciendo cambios muy rápidos en el mundo de la recuperación de información, desde el acceso a bases de datos con 
modems muy lentos (300 baudios) e interfaces de usuarios de línea de órdenes, hasta hoy día con velocidades de acceso decenas de veces más rápidas y con interfaces de usuario de tipo Web. Pero, ¿qué es lo que realmente ha cambiado? Analizaremos algunos distribuidores de información — hosts- y comprobemos si los cambios realizados han mejorado o no la pertinencia y exhaustividad de los documentos recuperados, ya que ese debería ser el principal objetivo de la recuperación de información. A continuación se describen los interfaces de distintos proveedores de bases de datos, los mas punteros en el mundo de la recuperación de información, con los que se pretende, evidentemente, facilitar al usuario final el acceso a la información.

\section{OCLC}

Online Computer Library Center, Inc, (OCLC) es una organización sin ánimo de lucro dedicada a la investigación y a la automatización de bibliotecas, que ofrece acceso público a información de todo el mundo a un coste reducido. Se puede acceder a su bases de datos por telnet (en desuso) o bien por web en la dirección <http://firstsearch.oclc.org>.

Actualmente el acceso está en proceso de cambio, pero las diferencias con el sistema anterior son escasas (http://newfirstsearch.oclc.org). La interfaz que presenta puede verse en la figura 1, a la que se accede después de introducir el mombre de usuario y la contraseña que nos identifica como usuario registrado en el sistema, y para que luego pueda facturarnos por las consultas realizadas. Un aspecto que hace más agradable la interfaz es que en http://newfirstsearch. oclc.org se puede seleccionar el idioma español para la interfaz de búsqueda, lo que hace más cómoda la utilización por parte de los hispano hablantes. En la figura 1 se puede ver cómo el sistema, la interfaz, permite dos opciones: a) puede sugerir las bases de datos adecuadas en función de las palabras que proporcione-

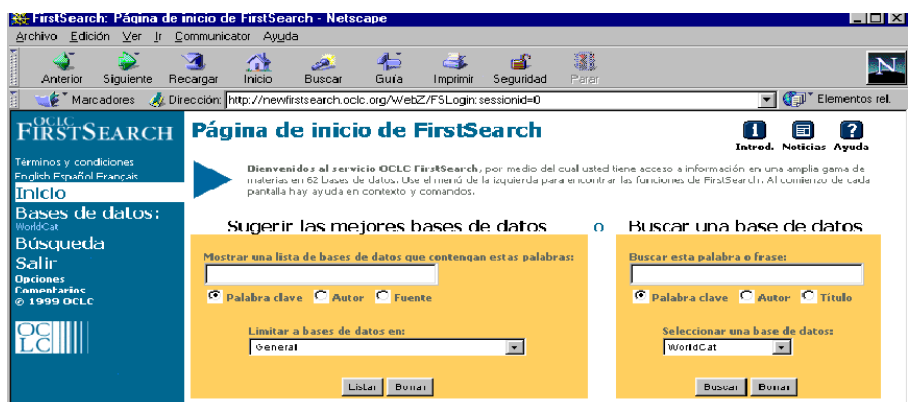

Figura 1. OCLC. Página de inicio de First Search

Scire. $7: 1$ (ene.-jun. 2001) 127-142. 
mos - palabras clave, autor o fuente-; y b) permite buscar la palabra o frase en la base de datos seleccionada de una lista desplegable. Es un sistema muy amigable para el usuario inexperto. Por medio de lenguaje natural puede ejecutar la búsqueda de información: No necesita saber ni las bases de datos necesarias, ni ningún comando de búsqueda para realizarla.

Cuando se desconocen las bases de datos más apropiadas para un tema, OCLC permite un sistema muy sencillo. Por ejemplo, si seleccionamos Lista de bases de datos por Tema, elegimos en Temas la opción General y realizamos la pregunta "cajal" como autor, el sistema OCLC muestra la relación de bases de datos que contienen información sobre el tópico utilizado. Las bases de datos están ordenadas de mayor a menor en los resultados, con una breve descripción de la temática de cada una de las bases de datos (figura 2) y se ofrece la posibilidad de seleccionar aquellas bases de datos en las que se desea realizar la consulta definitiva. En la selección de las bases de datos apropiadas sólo es posible buscar palabras clave, autor o fuente.

En el caso de haber elegido la base de datos, el tipo de búsqueda avanzada se realiza de la siguiente manera (figura 3): Se pueden introducir tres condiciones de búsqueda, con posibilidad de restringirlas a alguno de los campos de la base de datos que se haya seleccionado (autor, frase de autor, nombre de congreso, descriptor, etc). Estas condiciones pueden enlazarse con los operadores booleanos AND, OR, NOT. Además se pueden limitar los resultados de la búsqueda por años, tipo de documento, idioma, etc. Los resultados tambien se pueden ordenar

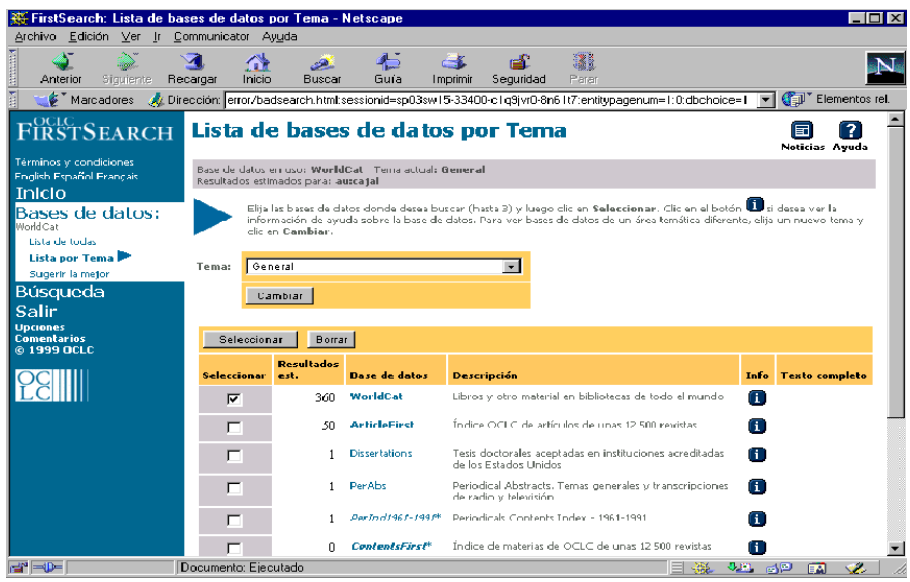

Figura 2. OCLC. Lista de bases de datos por tema

Scire. $7: 1$ (ene.-jun. 2001) 127-142. 


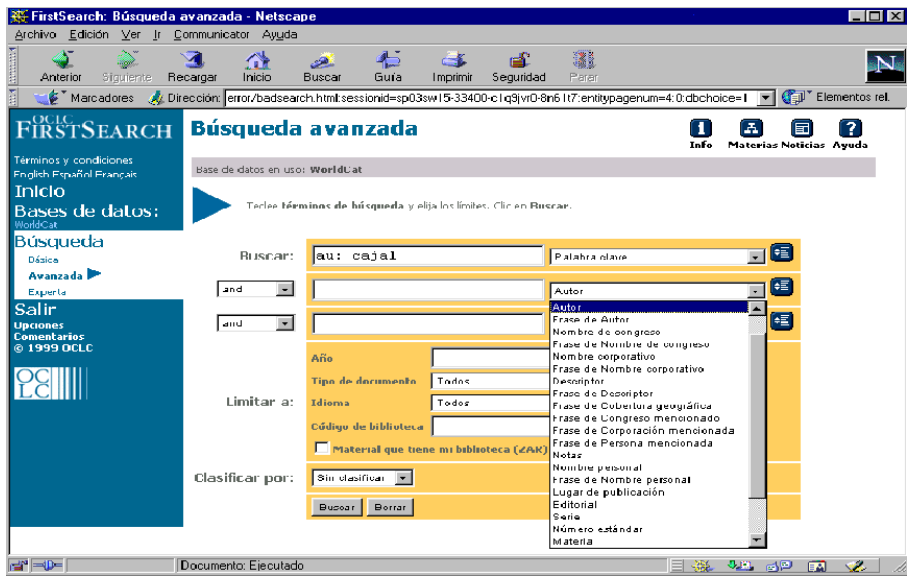

Figura 3. OCLC: Búsqueda avanzada

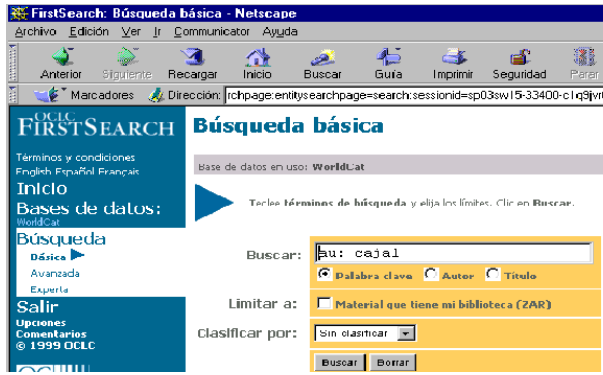

Figura 4. OCLC: Búsqueda básica

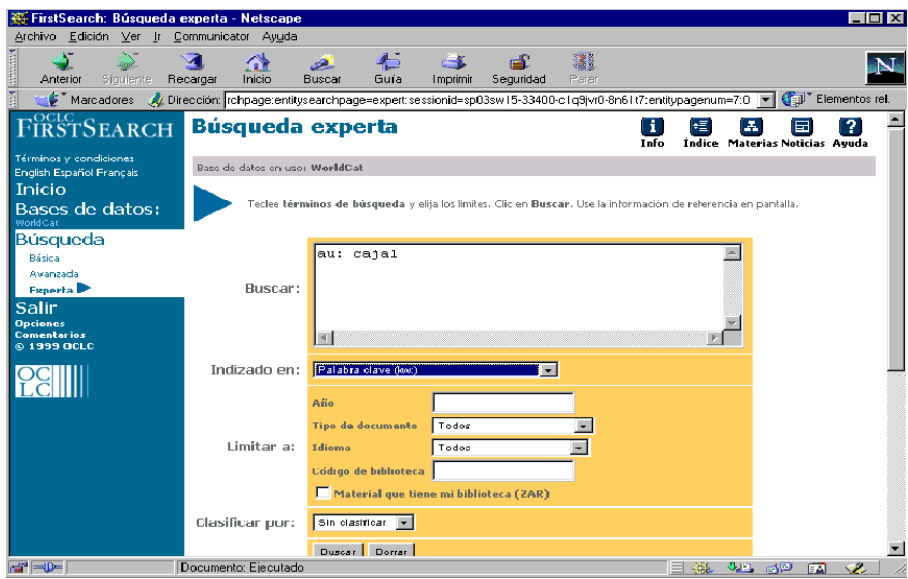

Figura 5: OCLC: Búsqueda experta

Scire. $7: 1$ (ene.-jun. 2001) 127-142. 
por el campo que se desee. El sistema OCLC da opción de realizar una búsqueda básica o una experta.

La búsqueda básica es una interfaz simple (figura 4) en la que sólo se pueden realizar determinadas preguntas: búsqueda por palabra clave, autor o título; limitar al material de nuestra biblioteca; y clasificar los resultados. Es el tipo de búsqueda que elegiría un usuario inexperto y que desea obtener resultados rápidos.

En cambio, la búsqueda experta (figura 5) permite un mayor control sobre cómo plantear la ecuación de búsqueda y, por lo tanto, sobre los resultados que se obtendrán, probablemente más pertinentes que con la búsqueda básica. Con esta interfaz de usurio hay que conocer el lenguaje de recuperación ya que en la casilla Buscar: hay que introducir las órdenes propias del lenguaje de recuperación empleado por OCLC. En la propia pantalla aparece un icono de ayuda que informa sobre cómo realizar la pregunta de manera correcta. A pesar de tener una interfaz de usuario gráfica, el sistema no ha sacrificado nada de la potencia del lenguaje de recuperación, es decir, respecto de una interfaz de línea de órdenes no se ha simplifacado casi nada el planteamiento de la estrategia de búsqueda.

\section{Dialog}

The Dialog Corporation, una compañía de Thomson, es el principal proveedor de información y de soluciones tecnológicas basadas en Internet para las empresas. Es el lider mundial de servicios en línea. Sus productos incluyen a DialoG, DataStar y Profound, que proporcionan acceso instantáneo a nueve terabytes —más de 6.000 millones de páginas— de información elaborada. Da servicios a unos 20.000 usuarios de 120 países.

Dialog ofrece dos niveles de búsqueda: usuario final y especialista en recuperación de información. El acceso a los servicios de Dialog se pueden hacer de distintas formas:

1. Acceso clásico por modem (Iberpac o RTC) y lenguaje de interrogación a las bases de datos con las órdenes propias del lenguaje de Dialog, introducidas en una línea de tipo MS-DOS.

2. Acceso clásico por Internet, mediante el lenguaje de interrogación clásico en línea de órdenes, ya sea con Telnet (telnet://dialog.com) o web (http://www.dialogclassic.com).

3. Internet con interfaz gráfica de usuario en entorno Web, con Dialog Web (http://www.dialogweb.com/), Dialog Select (http://products.dialog.com/ products/dialogselect/) o Dialog Portal (http://powerportal.dialog.com/).

La recuperación de información a través de interfaz clásica en Web, es idéntica a la realizada por métodos tradicionales. El único cambio que se observa es 
que la interfaz es gráfica. En lugar de la línea de órdenes típica, aparece una caja, tipo formulario, en la que, como se hace en la tradicional, se introduce la secuencia de órdenes (figura 6). La potencia del lenguaje es la misma, por lo que el usuario tiene que ser preferentemente un experto. La interfaz DialogWeb supone un cambio radical en la filosofía de búsqueda que hasta ese momento venía ofreciendo Dialog. En ella se aplica la tecnología cliente/servidor con una interfaz similar a la de OCLC, que permite seleccionar las bases de datos por materias y llegar a una pantalla con el formulario de búsqueda (figura 7).

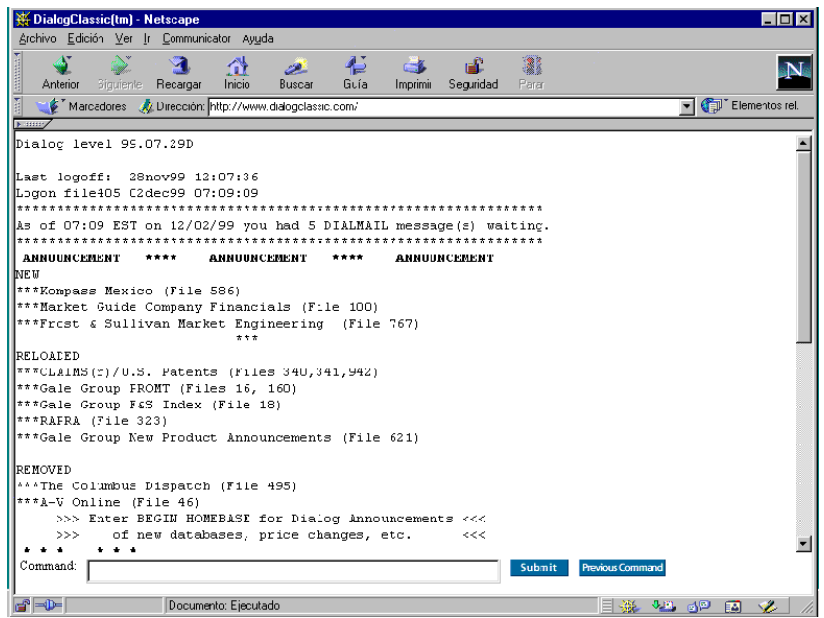

Figura 6. Dialog: Interface de DialogClassic

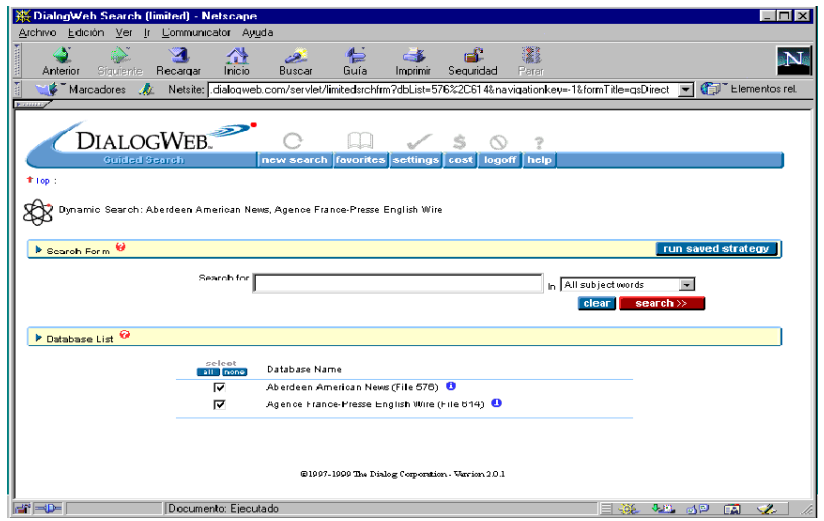

Figura 7. Dialog: Interface DialogWeb

Scire. $7: 1$ (ene.-jun. 2001) 127-142. 
Una vez introducida la pregunta y seleccionado el campo de la base de datos en el que se buscará, el sistema realiza la consulta presentando el título de los documentos encontrados en grupos de diez. A la vista de esa información, se pueden elegir los más apropiados pinchando en la casilla que aparece a la izquierda del título. Serán esos los documentos que Dialog cobre, al precio que se indica a la derecha, puesto que la visualización de los títulos es gratuita.

La interfaz DialogWeb va dirigida al usuario final, el cual no necesita ningún conocimiento sobre técnicas de recuperación, pero el sistema ha sacrificado, en aras a la sencillez de manejo, todos los elementos que permiten a los usuarios expertos localizar la información más pertinente.

El siguiente producto analizado son los portales de Dialog (Dialog Portals), que contienen sectores verticales ue integran información interna de The Dialog Corporation, con información extraída de otras fuentes, como, por ejemplo, páginas web profesionales actualizadas constantemente. Los tres portales que ofrece Dialog son: Negocios, Ciencia y Tecnología. Una vez que el usuario se ha registrado en el sistema, puede seleccionar el tema de búsqueda de entre los siguientes: Negocios y Noticias, Química, Energía y Medio Ambiente, Agricultura y Alimentación, Gobierno, Propiedad Intelectual, Medicina, Farmacia, Directorio y referencia, y Tecnología

\subsection{Lo mejor del Web}

Por ejemplo, en el caso de seleccionar el grupo de Propiedad Industrial (figura 8), se pueden elegir distintas búsquedas, en el lado izquierdo de la pantalla dentro del grupo de patentes: estado (status), reasignamiento (reassign) y documentos (documents). Después de seleccionar el grupo de bases de datos, la interfaz

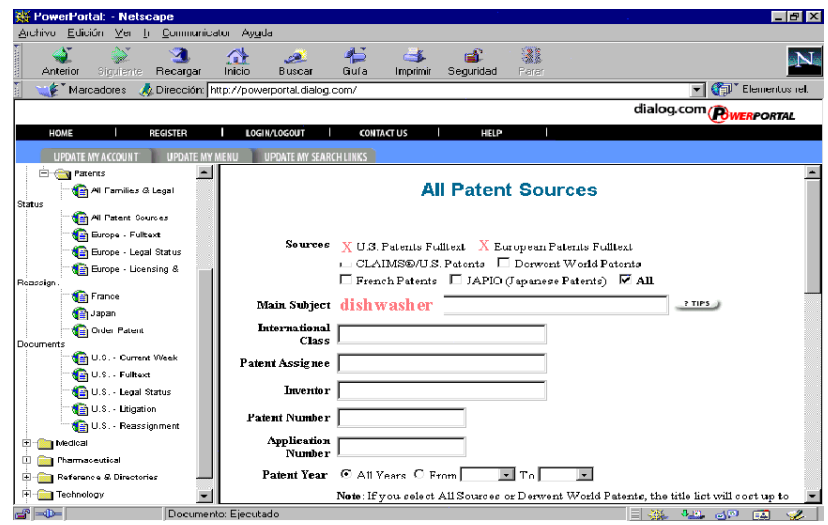

Figura 8. Dialog: Interface Dialog Portals

Scire. $7: 1$ (ene.-jun. 2001) 127-142. 
permite elegir todas o algunas de las bases de datos propuestas. En el ejemplo mostrado en la figura 8 , se han elegido por medio de un click de ratón en la casilla correspondiente, dos bases de datos: US Patents Fulltext y European Patents Fulltext. Además, se puede introducir el tema a buscar, dishwasher, la clasificación internacional, la empresa propietaria de la patente, el inventor, número de patente, número de aplicación y los años. Todas estas características permiten realizar una búsqueda bastante precisa, teniendo en cuenta que cada condición que añadamos se combinará con el operador booleano AND a las ya exigidas.

Una vez planteada la búsqueda, al pedir su ejecución, el sistema solicita el nombre de usuario y la contraseña para identificar al usuario. Seguidamente, el sistema devuelve los resultados mostrando los títulos de los documentos, que se pueden seleccionar con un click de ratón en la caja situada en la izquierda del documento para su visualización o impresión en formato completo. El sistema, avisa del coste de cada uno de los registros a la derech). También se puede realizar un Alert con la estrategia de búsqueda planteada, para recibir por email los nuevos documentos cada vez que se actualice la base de datos. Esta interfaz es bastante sencilla de utilizar por parte del usuario final, siempre que conozca las patentes y la terminología usada. Algunos tipos de búsquedas complejas no se pueden realizar con esta interfaz, por lo que habrá que recurrir a la búsqueda clásica, utilizando, por ejemplo, Dialog Classic.

Otro producto analizado es Dialog Select, que consiste en una selección de 250 bases de datos utilizables por el usuario final. Tras la selección de la materia por parte del usuario, el sistema elige las bases de datos en las que se realizará la

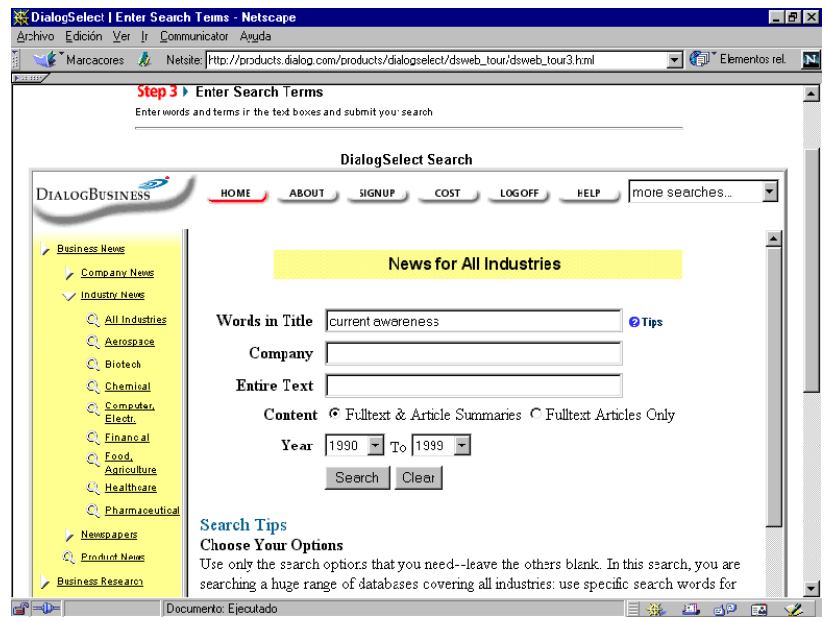

Figura 9: Dialog. Interface de DialogSelect

Scire. $7: 1$ (ene.-jun. 2001) 127-142. 
consulta. La interfaz es similar a la de Dialog Portals, pudiendo seleccionar, en el primer paso, un tema de entre los ofrecidos: DialogAgro, DialogBussiness, DialogChem, DialogEnergy, DialogGov, DialogIP (Intellectual Property), DialogMed, DialogNews, DialogPharma, DialogReference y DialogTech. El segundo paso es la selección de un tópico. Por ejemplo, si se ha elegido la categoría DialogBusiness (negocios), los tópicos que se pueden elegir son: Business News, Business Research, Company Financials, Company Products Line, Demographic Data (USA). En el ejemplo de la figura 9, se muestra el tercer paso: la introducción de los términos de búsqueda, concretamente en el apartado Business News. Aquí se pueden introducir las palabras del título, la compañía o del texto del documento. También se puede limitar la búsqueda a documentos en texto completo o texto completo y referencias; y por años de publicación. De manera análoga a DialogPortal, el sistema identifica al usuario y muestra los resultados agrupando los títulos de los documentos por decenas. No se observa ninguna diferencia esencial entre DialogPortal y DialogSelect, tal vez se puede precisar menos la búsqueda en este último.

Las tres últimas aplicaciones de Dialog que comentaremos brevemente, son: Intranet Toolkit, Intranet Delivery Tool, y Discovery.

Intranet Toolkit permite integrar los resultados de búsquedas en Dialog conjuntamente con la información interna y otras fuentes de la Intranet Corporativa. Permite compartir la información en toda la organización con un diseño personalizado de la interfaz de usuario. Es una aplicación muy util para el control del suministro de la información en toda la organización por parte del documentalista. El profesional de la información diseña el formulario de búsqueda adecuado para los usuarios de su organización, eligiendo las bases de datos en las que se buscará, las opciones de búsqueda y el formato de salida de las referencias encontradas. La aplicación proporciona las herramientas necesarias para realizar el diseño de la interfaz, bien totalmente original o bien partiendo de los 200 formularios ya creados por buscadores expertos y profesionales de la información. El acceso al Toolkit se realiza a través de contraseña en http://toolkit.dialog.com. En resumen, Dialog Intranet Toolkit está basado en una idea simple: permitir a los cliene de las bases de datos de Dialog construir sus propias interfaces para realizar las búsquedas.

Intranet Delivery Tool es una aplicación que permite que los resultados de los Alerts aparezcan directamente en la Intranet. Es decir, permite a los gestores del conocimiento crear en la Intranet sitios web personalizados para la recepción de los resultados de búsqueda o de Alerts. Una vez se han establecido las estrategias para alimentar la Intranet con la información requerida, la información obtenida estará a disposición de toda la empresa. El profesional de la información determina, conjuntamente con otros profesionales de la empresa, los contenidos que estarán presentes en la Intranet. El envío de los resultados de búsqueda o de 
los Alerts se realiza por correo electrónico. Esta aplicación, que deberá instalarse por el administrador del web, puede utilizarse para construir y organizar páginas web. Se pueden utilizar los comandos de búsqueda de Dialog y la Intranet Delivery Tool buscará exactamente la información solicitada y la distribuirá a los sitios intranet apropiados.

Discovery es una aplicación que permite recuperar información de forma intuitiva, con lenguaje natural. Los usuarios pueden buscar información en múltiples formatos de datos de manera transparente. Incluso si el usuario no es capaz de especificar sus intereses adecuadamente, Discovery establece un ambiente interactivo para ayudarle a localizar con precisión la información más relevante. Esta aplicación utiliza tecnología de inferencia lingüística para obtener resultados de alta calidad, sugiriendo nuevos conceptos y términos que mejoren su comprensión de sus áreas de interés. Los usuarios interactúan con un medio en el que se puede usar un auténtico lenguaje natural, de manera que no necesitan aprender ni usar sintáxis booleana. Los usuarios definen y redefinen sus intereses, recuperando diferentes tipos de información de diversas fuentes. Además, su interfaz permite integrar el tesauro de la organización. La British Library utiliza Discovery para ofrecer un acceso sencillo a sus amplísimas colecciones, sus páginas web y su intranet corporativa.

\section{STN}

STN International es el servicio de información online científico-técnico dedicado a cubrir las necesidades de información de científicos, ingenieros, y profesionales de la información de todo el mundo. STN proporciona una colección de bases de datos muy completa en las áreas de ciencia y tecnología dando acceso directo a la literatura, las patentes y los catálogos químicos. STN está dirigida por el Chemical Abstracts Service (CAS), una división de la American Chemical Society (USA), por FIZ Karlsruhe (Europa) y por la Japan Science and Technology Corporation, Information Center for Science and Technology (Japón).

El acceso a los servicios de STN se pueden hacer de forma clásica por modem (Iberpac o RTC) y lenguaje de interrogación a las bases de datos con los comandos propios del lenguaje de STN, introducidos en una línea de tipo MS-DOS. También por Internet, por medio del lenguaje de interrogación clásico en línea de órdenes, tanto mediante Telnet (telnet://141.166.16.239), Easy Web (http://www.fiz-karlsruhe.de/stneasy.html) o STN on the Web (http://stnweb.fiz-karlsruhe.de/).

Además ofrece un sistema de búsqueda realmente sencillo de usar, llamado STNEasy, es realmente sencilla de utilizar (figura 10). En dos pasos se puede realizar una consulta muy digna. El primer paso permite la selección de las bases de datos de forma temática. STN proporciona las siguientes categorías (32), a cada 


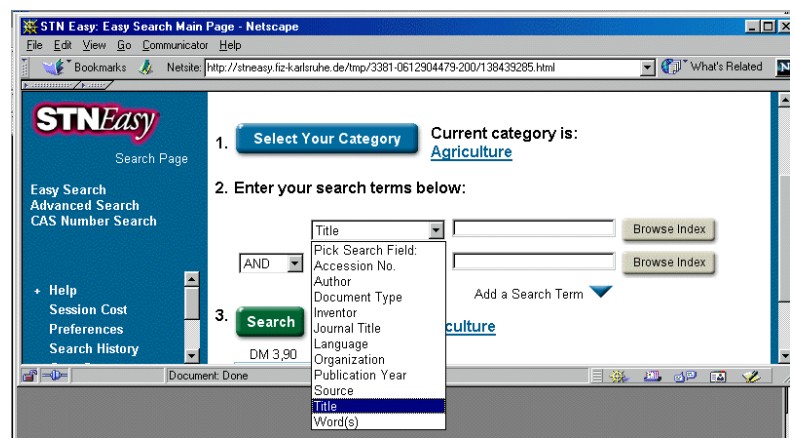

Figura 10. STN: Interface de STNEasy: selección del campo

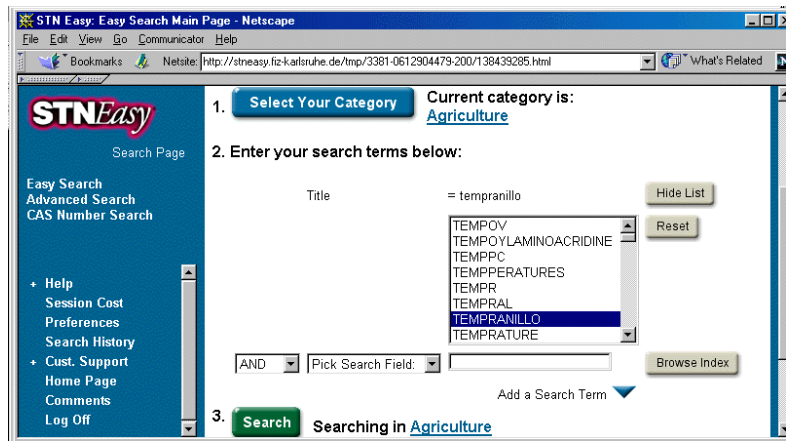

Figura 11. STN: Interface de STNEasy:consulta del índice

uno de ellos le corresponde una o más bases de datos: Agriculture; Biotechnology; Building \& Construction; Business; Chemical, Catalogs; Chemical, Substances; Chemistry, References; Chemistry, Analytical; Drug Names; Drug News; Energy; Engineering; Engineering, Chemical; Food; General Science; Life Sciences; Material Safety(MSDS); Materials; Materials, Polymers; Math/Computer Science; Patents; Patents, Citations; Patents, Fulltext; Patents, International; Patents, National; Petroleum; Pharmaceuticals; Pharmacology; Physics; Regulated Chemical Lists; Regulations, Government; y Toxicology. Seleccionada la categoría, el siguiente paso es la introducción de los términos de búsqueda. Esta interfaz, en este punto, presenta varias novedades interesantes. En primer lugar, permite visualizar la existencia del término, ya que STN ofrece el índice de palabras pulsando el botón "Browse Index" (figura 10). También permite elegir el campo en el que se desea realizar la búsqueda (figura 11) entre los siguientes: número de acceso, autor, tipo documento, inventor, título de la revista, idioma, organización, año de publicación, título, palabras. Con STNEasy también se pueden poner varias condiciones en la sentencia de búsqueda. Por defecto, ofrece la

Scire. $7: 1$ (ene.-jun. 2001) 127-142. 


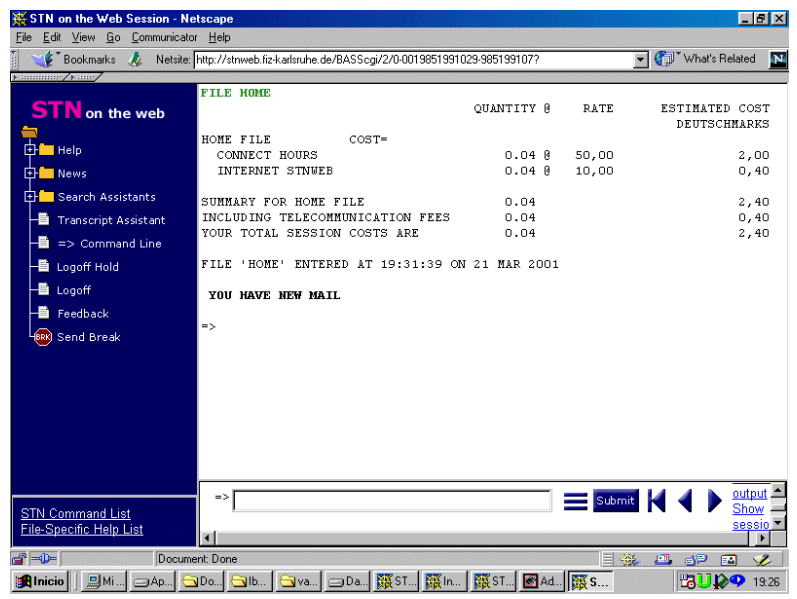

Figura 12. STN. Interface de STN on the Web

posibilidad de introducir dos condiciones, pero proporciona el enlace Add a search term, con lo que de hecho se pueden introducir todas las líneas de condiciones que se deseen con los operadores AND, OR, NEAR o NOT. De esa manera, la búsqueda es realmente flexible. Los resultados que se obtienen son similares a los de Dialog: un listado de títulos con los costes de cada uno de ellos en el caso de que se deseen obtener completos. STNEasy es una de las mejores interfaces que hemos encontrado, mezclando sencillez para el usuario final y bastante potencia para el usuario experto.

A diferencia de STNeasy, STN on the Web está pensado para los usuarios expertos. La Interfaz (figura 12) presenta un prompt, $=>$, igual que el de la línea de comandos tradicional, y por lo tanto hay que conocer los comandos del sistema para poder realizar la búsqueda. Hay una diferencia respecto al modo tradicional, y es que a la izquierda de la pantalla, STN on the Web ofrece una serie de ayudas, el Search Assistant, a las que se puede recurrir en caso necesario y que son más comodas que el correspondiente comando HELP en la línea de búsqueda.

\section{BookWhere}

Es un software que permite a los usuarios buscar en bases de datos de todo el mundo a través de Internet que cumplan los requisitos especificados en la norma Z39.50. La empresa tiene su sede en http://www.bookwhere.com/. El programa Bookwhere 2000 se instala en nuestro equipo y actua como cliente en una estructura de tipo cliente/servidor. La utilización es muy intuitiva para el usuario final, pero hay que realizar algunos cambios para que funcione según nuestras prefe- 
rencias. Se configura, en primer lugar, las bases de datos a las que se desea acceder. Inicialmente, por defecto, el programa ofrece varios cientos de OPACS (Online Public Access Catalogs) de todo el mundo a los que se puede acceder para buscar la información requerida. En la figura 13 se puede observar la interfaz que permite seleccionar las bases de datos, las cuales están organizadas por países. Además, se pueden añadir nuestras preferidas, generalmente los catálogos de las bibliotecas nacionales u otras más útiles para nosotros. Seleccionadas las bases de datos, el software ofrece dos posibilidades para plantear la estrategia de búsqueda: sencilla o compleja (simple / power).

La búsqueda sencilla (figura 14) consiste en rellenar en el formulario presentado los campos autor, título, materia o texto libre. Además, incluye la posibili-

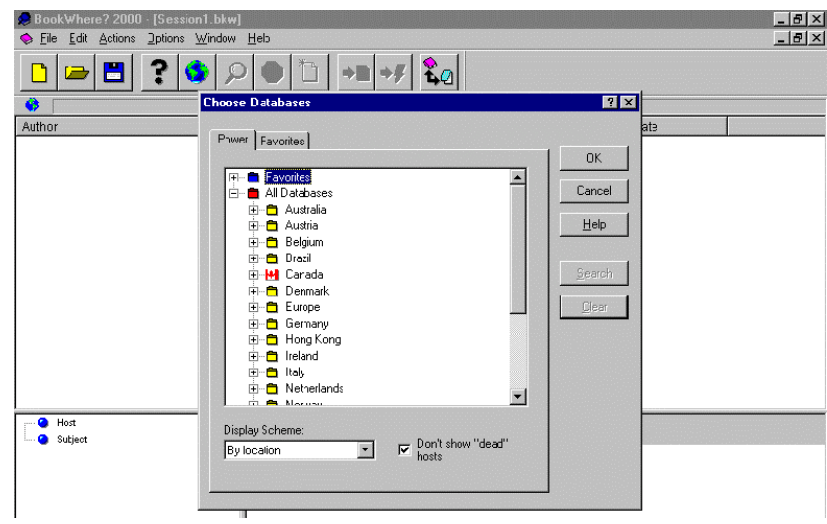

Figura 13. Interfaz para la selección de las bases de datosen BookWhere

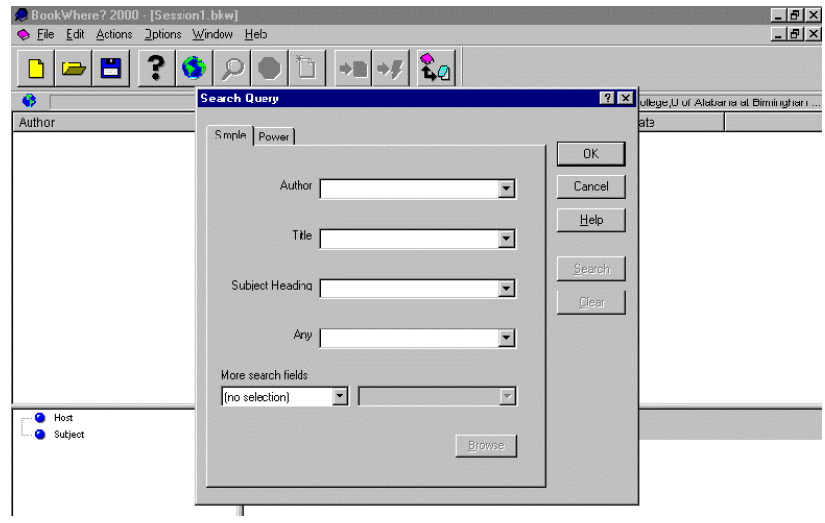

Figura 14. BookWhere. Interface de búsqueda sencilla

Scire. $7: 1$ (ene.-jun. 2001) 127-142. 
dad de añadir otros campos en More search fields, lo que permite hacer una búsqueda bastante eficiente y controlable por el usuario final. La búsqueda compleja (figura 15) presenta una interfaz con las mismas posibilidades que la sencilla (autor, título, materia o texto libre) pero ahora cada una de las opciones se puede expandir para hacerla tan compleja como se necesite por medio de operadores de relación, truncado, estructura, posición y completitud. Es decir, la complejidad aumenta, aunque el usuario final no hallará ninguna dificultad en manejarse y plantear la búsqueda.

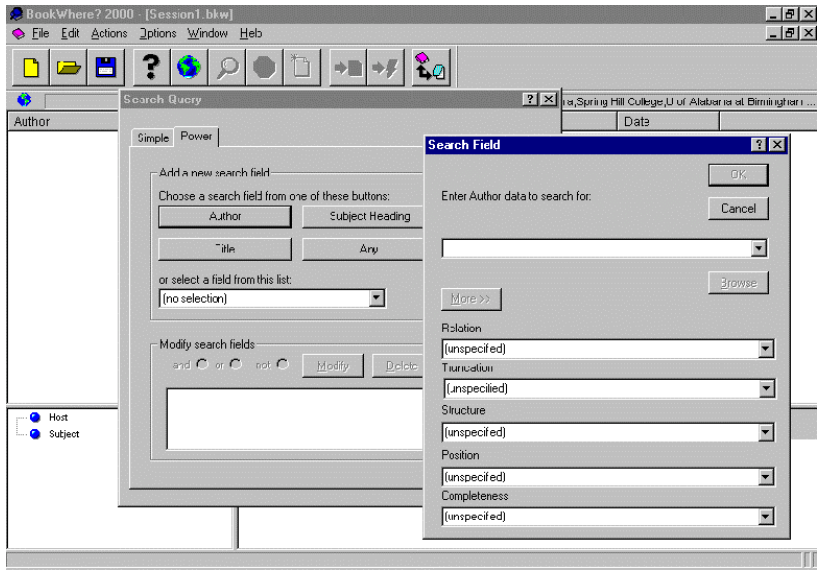

Figura 15. BookWhere. Interface de búsqueda compleja

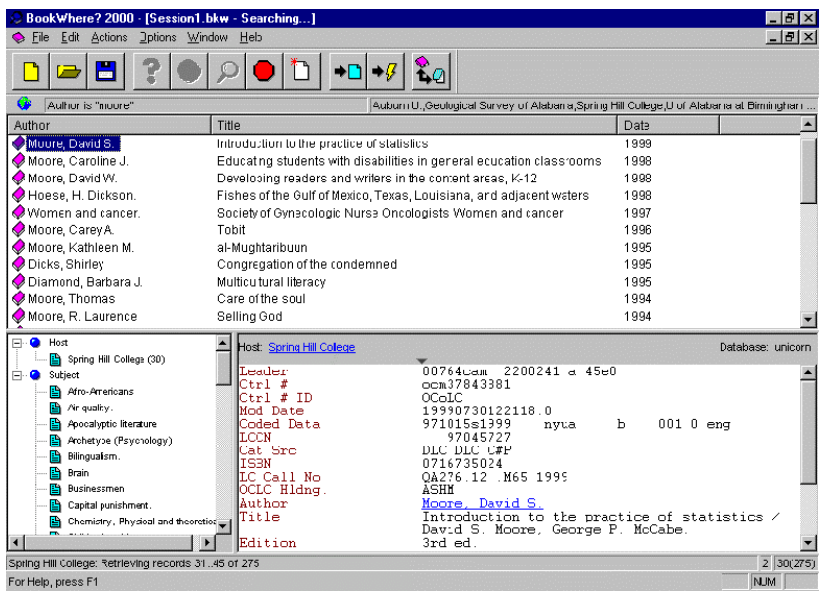

Figura 16. BookWhere: Resultados de una búsqueda

Scire. $7: 1$ (ene.-jun. 2001) 127-142. 
Los resultados que se obtienen se muestran en la figura 16. La gran ventaja de que distintas bases de datos utilicen la norma Z39.50 es que todas ellas se pueden interrogar con la misma interfaz y que los resultados se presentan de manera uniforme. En este ejemplo puede verse en la parte superior la información abreviada de los registros encontrados — autor, título y fecha de publicación-; en la parte inferior izquierda está la información de la biblioteca en la que se ha encontrado el registro seleccionado; y en la parte inferior derecha se muestra el registro en formato completo. Además, en la pantalla aparecen algunos datos más: los registros encontrados en ese host, Spring Hill College, los registros mostrados, etc.

\section{Conclusiones}

Hemos comprobado exhaustivamente las grandes mejoras que todos los proveedores de bases de datos han hecho en lo últimos años. Pero, ¿qué han mejorado realmente?: los interfaces de búsqueda, dirigidos al usuario final. Todos los hosts adoptan las mismas o parecidas soluciones: browser en Internet con un formulario de búsqueda, en general sencillo. Algunos, como Dialog y STN, permiten la realización de la búsqueda y "sólo" cobran cuando vemos las referencias completas, indicándonos amablemente el coste de cada una de ellas.

En este camino se han ido perdiendo las grandes posibilidades ofrecían los lenguajes de recuperación de modo comando, que permiten a los usuarios expertos una búsqueda "más fina”. Sólo la aplicación SciFinder, creada por el Chemical Abstracts Service, ha conservado todas las prestaciones para las búsquedas en el campo de la química, pero con una utilización simple y una capacidad de búsqueda impresionante. Ahora bien, el usuario final deberá ser un experto en Química, o como mínimo con ámplios conocimientos sobre ella, y deberá realizar un entrenamiento que le permita sacar el máximo provecho. En definitiva, tampoco es para el usuario final en general, sino especialista en la materia.

Por otra parte, se está abriendo poco a poco camino la posibilidad de utilizar el lenguaje natural en la interrogación a bases de datos. Como en casi todas las ocasiones, el host Dialog lleva ventaja con herramientas como Discovery.

La posición del profesional de la información está cambiando, aunque sigue siendo muy importante, especialmente en la implantación de herramientas como BookWhere, Intranet Toolkit o Intranet Delivery Tool. La puesta a punto de las interfaces que el personal de su organización tienen que utilizar es una responsabilidad que debe asumir, permitiendo al usuario final el acceso directo a las consultas de bases de datos, con la garantía de que la búsqueda se realizará con calidad y precisión, independientemente de las habilidades de cada uno de los usuarios finales de su empresa.

Scire. $7: 1$ (ene.-jun. 2001) 127-142. 


\section{Bibliografía}

Galitz, W. O. (1996). The Essential Guide to User Interface Design: An Introduction to Gui Design Principles and Techniques. New York: John Wiley \& Sons. 1996.

Hu, P. J. H. ; Ma, P. C. ; Chau, P. Y. K. (1999). Evaluation of user interface designs for information retrieval systems: a computer-based experiment. // Decision Supports Systems. 27 : 1-2 (1999) 125-143.

Nielsen, J. ; Del Galdo, E.M. (1996). International User Interfaces. New York : John Wiley \& Sons, 1996.

Nielsen, J. (1999). User interface directions for the Web. // Communications of the ACM. $42: 1$ (1999) 65-72.

Reed, P. ; Holdaway, K. ; Isensee, S. ; Buie, E. ; Fox, J. ; Williams, J. ; Lund, A. (1999). User interface guidelines and standards: progress, issues, and prospects. // Full source Interacting with Computers. 12 : 2 (1999) 119-142.

The Dialog Corporation (2000). Dialog Intranet Toolkit Instructional Guide. Actualizado: 05/09/2000. URL: <http://training.dialog.com/cip-lis/pdf/toolkit_guide.pdf>. Fecha de consulta: 15-03-2001)

The Dialog Corporation (2000). How the British Library Uses k-working Discovery to Offer Easy Access to Its Vast Collection. Chronolog, $3 / 4$ (2000). URL: <http://library.dialog.com/chron/2000/0003/1000328.html>. Fecha de consulta: 1503-2001.

The Dialog Corporation (1999). Intranet Delivery Tool: Major Enhancement to Dialog Intranet Toolkit Announced. Chronolog, May/June 1999. URL: <http://library.dialog.com/chron/1999/9905/990538.html>. Fecha de consulta: 15-03-2001. 